\title{
Genetic Role of BDNF Val66Met and 5-HTTLPR Polymorphisms on Depressive Disorder
}

\author{
Kyu Young Lee ${ }^{1,2}$, Seong Hoon Jeong, ${ }^{1,3}$, Se Hyun Kim4, Yong Min Ahn ${ }^{4,5,6,7}$, Yong Sik Kim², \\ Hee Yeon Jung ${ }^{5,9}$, Yang Weon Bang ${ }^{10}$, and Eun-Jeong Joo ${ }^{1,2} \bowtie$ \\ ${ }^{1}$ Department of Neuropsychiatry, Eulji University School of Medicine, Daejeon, Republic of Korea \\ ${ }^{2}$ Department of Psychiatry, Eulji General Hospital, Seoul, Republic of Korea \\ ${ }^{3}$ Department of Psychiatry, Eulji University Hospital, Daejeon, Republic of Korea \\ ${ }^{4}$ Institute of Human Behavioral Medicine, Medical Research Institute, Seoul National University, Seoul, Republic of Korea \\ ${ }^{5}$ Department of Psychiatry and Behavioral Science, Seoul National University College of Medicine, Seoul, Republic of Korea \\ ${ }^{6}$ Department of Neuropsychiatry, Seoul National University Hospital, Seoul, Republic of Korea \\ ${ }^{7}$ Biomedical Research Institute, Seoul National University Hospital, Seoul, Republic of Korea \\ ${ }^{8}$ Department of Neuropsychiatry, Dongguk University International Hospital, Dongguk University Medical School, Goyang, Republic of Korea \\ ${ }^{9}$ Department of Psychiatry, SMG-SNU Boramae Medical Center, Seoul, Republic of Korea \\ ${ }^{10}$ Department of Psychiatry, Keyo Hospital, Uiwang, Republic of Korea
}

\begin{abstract}
Objective We investigated possible association between depressive disorders and BDNF Val66Met and 5-HTTLPR. Brain derived neurotrophic factor (BDNF) gene and serotonin transporter (SLC6A4) gene are promising candidate genes for depressive disorders. It has been suggested that BDNF promotes the survival and differentiation of serotonergic neurons and that serotonergic transmission exerts powerful control over BDNF gene expression.

Methods Final analyses were performed on 186 patients with depressive disorders and 1032 controls. Val66Met polymorphism of BDNF gene and 5-HTTLPR polymorphism of serotonin transporter gene were genotyped and allele and genotypic associations on the diagnosis of depression and age at onset of depression were analyzed.

Results The 5-HTTLPR was positively associated with depressive affected status in the total sample and in females ( $\mathrm{p}=0.038$ for allelewise, $\mathrm{p}=0.015$ for genotype-wise associations), but, not in males. The BDNF Val66Met showed no association with depression. BDNF Val66Met and 5-HTTLPR alone were not associated with age at onset of depression. Additional analysis on the interaction between BDNF Val66Met and 5-HTTLPR found a significant association with age at onset of depression in the entire patient group. This association was also found in the female but not in the male patient group. None of the positive results survived Bonferroni correction for multiple testing.

Conclusion This result suggested that BDNF Val66Met and 5-HTTLPR may contribute to depressive disorders in a complex way and that the genetic effect could differ by gender. Further studies with large number of patients will be necessary.

Psychiatry Investig 2014;11(2):192-199
\end{abstract}

Key Words BDNF Val66Met, 5-HTTLPR, Depressive disorder, Genetic polymorphism, Onset, Serotonin transporter.

\section{INTRODUCTION}

Depressive disorder is a complex disease phenotype. Genet-

Received: May 10, 2013 Revised: June 28, 2013

Accepted: August 2, 2013 Available online: April 11, 2014

$\triangle$ Correspondence: Eun-Jeong Joo, $\mathrm{MD}, \mathrm{PhD}$

Department of Psychiatry, Eulji General Hospital, 68 Hangeulbiseok-ro, Nowon-gu, Seoul 139-711, Republic of Korea

Tel: +82-2-970-8611, Fax: +82-2-949-2356, E-mail: jej1303@gmail.com

(a) This is an Open Access article distributed under the terms of the Creative Commons Attribution Non-Commercial License (http://creativecommons.org/licenses/by$\mathrm{nc} / 3.0$ ) which permits unrestricted non-commercial use, distribution, and reproduction in any medium, provided the original work is properly cited. ic studies have indicated that genes work significantly as etiological factors and disease modifiers in depression. However, no major genes for depressive disorder likely exist. Accumulating genetic studies such as candidate gene studies, genomewide association studies, and rare-variant studies strongly suggest that polygenes with small effects and rare mutations are likely genetic precipitating factors. Among various promising candidate genes for depression, the serotonin transporter (SL$\mathrm{C} 6 \mathrm{~A} 4)$ and brain-derived neurotrophic factor (BDNF) genes are two of the most studied candidate genes for depressive disorders worldwide. 
The serotonin transporter-linked polymorphic region (5HTTLPR) for SLC6A4 is located upstream of the transcription initiation site of the SLC6A4 gene. 5HTTLPR is a 44-bp insertion/deletion variable-number tandem repeat polymorphism with two common alleles: the 16-repeats long allele and the 14-repeat short allele. 5HTTLPR is a functional polymorphism in that the short allele has lower transcriptional efficiency. ${ }^{1,2}$ Studies on 5-HTTLPR and depressive disorders including meta-analyses can be summarized as follows. First, the association with depression itself seems to be positive. Clarke et al. ${ }^{3}$ reported a small but statistically significant association based on a meta-analysis of 39 studies. Second, an association with the antidepressant response is less likely. Taylor et al. analyzed 28 studies and found no significant effect on remission rates after correcting for missing data. ${ }^{4}$ Third, the genetic moderation by 5 -HTTLPR of environmental factors for the development of depression is strongly supported. Earlier studies found a significant interaction between 5-HTTLPR and the number of life depressive events, and the short allele indicates a higher vulnerability to social stressors. This suggests that environmental insult is moderated by this gene. ${ }^{5,6}$ A meta-analysis including 14 studies found no evidence of an association between the serotonin-transporter genotype alone and no interaction with stressful life events. ${ }^{7}$ However, Karg et al. ${ }^{8}$ recently included 54 studies in a meta-analysis and found that the s allele was associated with an increased risk of depression under stress.

BDNF is one of the neurotrophic factors manifested in the brain and multiple tissues and has been suggested as a biomarker of depressive disorders. ${ }^{9}$ Findings indicating that BDNF levels are reduced in the postmortem brain and blood of patients with depressive disorders and that antidepressant treatments increase BDNF levels support the neurotrophic hypothesis for the etiology of depression. ${ }^{10,11}$ BDNF Val66Met (G196A, rs6265) polymorphism is located at an exon and changes an amino acid at codon 66 from Val to Met. Positive association studies have been published, ${ }^{12-14}$ however, whether the Met or Val allele is a risk allele for depression has not been confirmed. Frielingsdorf et al. reported that Met homozygotes may have a greater risk for major depressive disorder. ${ }^{14}$ Another study found that Val homozygotes have an increased chance of depression. ${ }^{13}$ However, negative association results have also been found, ${ }^{15,16}$ and a meta-analysis involving 14 studies reported that BDNF Val66Met was not significantly associated with depression in a total sample and that a significant effect was found only in men. ${ }^{17}$ Environmental interactions with this SNP that increase susceptibility for major depressive disorder after early-life stress may be associated with the Met allele. ${ }^{18}$ Taken together, these results indicate that the genetic effect of BDNF Val66Met on depression is not fully understood.

A significant interaction between BDNF and serotonin has been suggested in brain function. Based on a common feature of their regulatory ability for the development and plasticity of neural circuits, the interaction of these two systems is likely involved in mood disorders. BDNF promotes the survival and differentiation of serotonergic neurons, and serotonergic transmission exerts powerful control over BDNF gene expression. ${ }^{19}$ Administration of SSRIs positively regulates BDNF transcription through increases in CREB phosphorylation. ${ }^{20}$ Other evidence for this positive interaction is substantial. However, specific combinations of risk alleles are not consistent. The Met allele of the BDNF Val66Met polymorphism protects against deleterious effects of the 5-HTTLPR $s$ allele on brain volume. ${ }^{21}$ The Met allele seems to confer a better response to lithium in s-carrier patients. ${ }^{22}$ In contrast, the BDNF Met allele carriers and 5-HTTLPRs homozygotes have the highest depression vulnerability under adverse environments. ${ }^{23,24}$ Understanding the interactions between these two systems is crucial to help obtain a more comprehensive view of the molecular pathways of mood regulation. ${ }^{19}$

Age at onset is an important clinical feature of disease and may be influenced by genetic factors. Genetic factors for the age at onset could be identical to or different from the genetic factors in the diagnosis of depressive disorder. In the present study, we investigated a possible association between depressive disorder and BDNF Val66Met and 5-HTTLPR, which are promising candidate genetic polymorphisms in psychiatric genetics. The influences of individual genes and of the interaction between polymorphisms on the diagnosis of depression and age at onset were explored. The association between age at onset of depressive disorder and a genetic interaction between BDNF Val66Met and 5-HTTLPR has not been investigated previously. This study aimed to replicate the genetic association of BDNF Val66Met and 5-HTTLPR with depressive disorder in a Korean population and to explore the interactive genetic role of BDNF Val66Met and 5-HTTLPR on age at onset of depressive disorders.

\section{METHODS}

\section{Subjects}

Patients were recruited from Eulji General Hospital. All patients were evaluated based on the diagnostic criteria of the DSM-IV. We included a broad spectrum of depressive disorders for this study, i.e., major depressive disorder, dysthymia, and depressive disorder, not otherwise specified (NOS). Both single-episode and recurrent major depressive disorder were included, and major depressive disorder with dysthymia was also included. Patients were individually interviewed by re- 
search nurses using the Korean version of the Mini-International Neuropsychiatric Interview. Consensus diagnostic meetings of more than two psychiatrists, including the psychiatrist in charge of the psychiatric treatment of the patient, made the final diagnosis of participants. Associated symptoms and other clinical characteristics (Table 1) were collected based on patient-provided information. Psychotic features, psychomotor retardation, somatization, suicide attempts, panic symptoms, alcohol problems, and familial loading of major psychiatric disorders were evaluated by the psychiatrist in charge. Evaluation of weight gain, increased appetite, and increased sleep were largely based on subjective information from patients. Subjects with a history of any kind of organic abnormality of the brain, substance dependence, drug abuse, or other physical conditions possibly manifesting as psychiatric symptoms were excluded from this study. Controls were recruited from college students, nurses, and fire and public protection officers. A brief psychiatric interview was performed by psychiatric research nurses to evaluate current and past psychiatric illness. Subjects with a lifetime history of major psychiatric illness and/or brain trauma were excluded. Final analyses were performed on 186 patients and 1032 controls. The patient group consisted of 106 patients with recurrent major depressive disorder, 38 patients with a single episode of major depressive disorder, 17 patients of major depressive disorder with dysthymia, seven patients with dysthymia, and 19 patients with depressive disorder, NOS. Control subjects, matched for sex ratio with the patient group, were randomly selected from our control pool. The mean ages of the patient and control groups were $52.14 \pm 14.38$ and $24.43 \pm$ 5.07 years, respectively. More detailed information regarding the subject number and mean age for each group is given in Table 2. All subjects participating in this study signed a written informed consent form, and the study protocol was approved by the ethics committee of Eulji General Hospital.

\section{Genotyping}

DNA was extracted from blood samples using a DNA isolation kit (Roche, Mannheim, Germany). The Val66Met SNP of the BDNF gene and the 5-HTTLPR polymorphism of the serotonin transporter gene were genotyped. Genotyping for SNP Val66Met was performed using the TaqManTM method (Applied Biosystems, Foster City, CA, USA) ${ }^{25}$ Primers for the Val66Met G/A polymorphism were synthesized with the sequences 5'-TCAAGAGGCTTGACATCATTGG-3' for the forward primer and 5'-GCCGAACTTTCTGGTCCTCAT-3' for the reverse primer. FAM and VIC dye probes were also synthesized with the sequences 5'-6FAM-ACACTTTCGAA

Table 1. Clinical characteristics of patients with depression

\begin{tabular}{|c|c|c|c|c|c|}
\hline & \multirow{2}{*}{$\begin{array}{c}\text { Total } \\
\text { Number }\end{array}$} & \multicolumn{2}{|c|}{ Male } & \multicolumn{2}{|c|}{ Female } \\
\hline & & Number & $\%$ & Number & $\%$ \\
\hline \multicolumn{6}{|l|}{ Diagnosis } \\
\hline MDD signle & 37 & 11 & 28.21 & 26 & 17.69 \\
\hline MDD recurrent & 106 & 19 & 48.72 & 87 & 59.18 \\
\hline MDD with dysthymia & 17 & 3 & 7.69 & 14 & 9.52 \\
\hline Dysthymia & 7 & 3 & 7.69 & 4 & 2.72 \\
\hline Depressive disorder, NOS & 19 & 3 & 7.69 & 16 & 10.88 \\
\hline \multicolumn{6}{|l|}{ Associated symptom } \\
\hline Psychotic feature & 23 & 10 & 25.64 & 13 & 8.84 \\
\hline Psychomotor retardation & 56 & 14 & 35.90 & 42 & 28.57 \\
\hline Weight gain & 26 & 3 & 7.69 & 23 & 15.65 \\
\hline Increased appetite & 14 & 1 & 2.56 & 13 & 8.84 \\
\hline Increased sleep amount & 11 & 2 & 5.13 & 9 & 6.12 \\
\hline Somatization & 151 & 25 & 64.10 & 126 & 85.71 \\
\hline Suicidal attempt & 22 & 6 & 15.38 & 16 & 10.88 \\
\hline Panic symptom & 26 & 1 & 2.56 & 25 & 17.01 \\
\hline \multicolumn{6}{|l|}{ Characteristics } \\
\hline Alcohol problem & 8 & 6 & 15.38 & 2 & 1.36 \\
\hline Familial loading & 46 & 9 & 23.08 & 37 & 25.17 \\
\hline Postmenopausal status & & & & 87 & 59.18 \\
\hline
\end{tabular}

MDD: major depressive disorder, NOS: not otherwise specified 
CACGTGATAGAAGAGCTGTT-TAMRA-3' for the FAM dye probe and 5'-TET-ACACTTTCGAACACATGATAG AAGAGCTGTTG-TAMRA-3' for the VIC dye probe. Standard polymerase chain reaction (PCR) analyses was carried out in $5-\mu \mathrm{L}$ volumes that contained $50 \mathrm{ng}$ of genomic DNA, $2.5 \mu \mathrm{L}$ of $2 \times$ TaqMan Master Mix, $600 \mathrm{nM}$ of each primer, and $100 \mathrm{nM}$ of FAM and VIC dye probes. After an initial 2 minutes of denaturation at $50^{\circ} \mathrm{C}$ followed by 10 minutes at $95^{\circ} \mathrm{C}, 40$ thermal cycles consisting of 15 seconds at $95^{\circ} \mathrm{C}$ and 1 minute at $60^{\circ} \mathrm{C}$ were carried out. Automated reading was done with an ABI Prism 7900HT Sequence Detection System (Applied Biosystems). The 5-HTTLPR polymorphism was genotyped as follows. Primers for 5-HTTLPR were synthesized with the sequences for stpr5: 5'-GGCGTTGCC GCTCTGAATGC-3' and stpr3: 5'-GAGGGACTGAGCTG GACAACCAC-3'. A $1-\mu \mathrm{L}$ aliquot of $50 \mathrm{ng} / \mu \mathrm{L}$ DNA, $2 \mu \mathrm{L}$ of $20 \mathrm{mM}$ dNTP mix (Roche), $0.5 \mu \mathrm{L}$ of each $20 \mathrm{pM}$ primer, 1.5 $\mu \mathrm{L}$ of $10 \times$ buffer, $1.5 \mathrm{U}$ of Taq polymerase (Roche), and 9.2 $\mu \mathrm{L}$ of distilled water were mixed to make a $15-\mu \mathrm{L}$ PCR reaction volume. The PCR was carried out using the following steps: $5 \mathrm{~min}$ at $94^{\circ} \mathrm{C}$ to denature the DNA sequence, 35 cycles at $94^{\circ} \mathrm{C}$ for $30 \mathrm{sec}, 64^{\circ} \mathrm{C}$ for $30 \mathrm{sec}$, and $72^{\circ} \mathrm{C}$ for $1 \mathrm{~min}$, followed by $7 \mathrm{~min}$ for amplification at $72^{\circ} \mathrm{C}$. PCR products were examined by electrophoresis for $30 \mathrm{~min}$ on a $3 \%$ agarose gel stained with ethidium bromide. DNA sequencing of the PCR products for several subjects was performed to confirm the 5-HTTLPR genotype.

\section{Statistical analysis}

Hardy-Weinberg equilibrium was tested by a goodness-offit chi-square test. The entire analysis was conducted using UNPHASED 3.0.13 software (http://www.mrc-bsu.cam.ac. uk/personal/frank/software/unphased/). Allelic and genotypic associations of each polymorphism were tested for the association with diagnosis of depression. The program calculated chi-square values and p-values for allelic and genotypic interactions according to a full model. The level of statistical significance was set at $\mathrm{p}=0.05$. Permutation tests were performed 1000 times to avoid false discovery from initial analyses. Age at onset was tested for an association with each polymorphism by the chi-square test with UNPHASED 3.0.13 and analysis of variance (ANOVA). Three groups for BDNF Val66Met (Met/Met, Met/Val, and Val/Val) and two groups for 5-HTTLPR (ss and sl, and ll) were constructed for six combined-genotype groups. Analysis for a possible association between age at onset and an interaction of BDNF Val66Met with 5-HTTLPR was performed by ANOVA for these six groups. The ANOVA was performed using SPSS 12.0 software (Chicago, IL, USA).

\section{RESULTS}

The patient group consisted of 147 females and 39 males. As females were predominant in the patient group, we randomly selected control subjects under the condition of matching the sex ratio with the patient group. Major depressive disorder was the most common diagnosis in this patient group. Major depressive disorders constituted $77 \%$ of the entire patient group. About $60 \%$ of patients were recurrent major depression and their age and age at onset were not significantly different from the other patients. The age at onset was $44.02 \pm$ 15.32 years old for the total patient group and was not statistically different according to gender (Table 2). Somatization symptoms and panic symptoms associated with depressive disorder were found significantly more often in the female patient group ( $\mathrm{p}=0.005$ for somatization and $\mathrm{p}=0.019$ for panic symptoms; chi-square test). Associated psychotic features and alcohol problems were significantly more frequent in the male patient group ( $\mathrm{p}=0.011$ for psychotic features and $\mathrm{p}<0.001$ for alcohol problems, chi-square test). More than half of the female patients (59\%) were postmenopausal (Table 1).

The serotonin transporter 5-HTTLPR polymorphism was positively associated with depression. Both allele and genotype associations were significant in the total sample ( $\mathrm{p}=0.038$ for allele-wise, $\mathrm{p}=0.015$ for genotype-wise associations). When we conducted the analysis separately according to gender, a significant genetic association was found in the female group but not in the male group. The significance survived 1000 permutations for both the total and female groups (Table 3). The BDNF Val66Met SNP was not associated with depression (Table 4).

Age at onset as a quantitative phenotype was tested for a

Table 2. Age and age at onset of subjects

\begin{tabular}{|c|c|c|c|c|c|c|c|c|c|}
\hline & \multicolumn{3}{|c|}{ Depression } & \multicolumn{3}{|c|}{ Control } & \multicolumn{3}{|c|}{ Recurrent depression } \\
\hline & Total & Male & Female & Total & Male & Female & Total & Male & Female \\
\hline Number & 186 & 39 & 147 & 1032 & 219 & 813 & 106 & 19 & 87 \\
\hline Age & $52.14(14.38)$ & $52.67(17.73)$ & $52.00(13.42)$ & $24.43(5.07)$ & $27.10(8.15)$ & $23.71(3.52)$ & $54.87(11.90)$ & $54.74(17.36)$ & 54.90 \\
\hline Age at onset & $44.02(15.32)$ & $45.85(19.62)$ & 43.53 (13.99) & & & & $44.56(13.41)$ & $44.58(18.80)$ & 44.55 \\
\hline
\end{tabular}

Mean values for age and age at onset were presented. SD were pressented in parenthesis 
possible association with BDNF Val66Met, 5-HTTLPR, and the interaction of polymorphisms. Both the chi-square and ANOVA tests consistently found that neither BDNF Val66Met alone nor 5-HTTLPR alone was associated with age at onset of depressive disorder in our sample (Table 3 and 4). The Met/Met genotype of the BDNF Val66Met polymorphism was associated with the oldest age at onset (46.65 \pm 16.61 years), and the Val/Val genotype with the youngest age at onset (41.81 \pm 14.98 years). The Met allele tended to be associated with older age at onset in a dose-dependent manner, and this association was significant $(\mathrm{p}=0.293$ ) (Table 5). Both the ss genotype and sl genotype of the 5-HTTLPR polymorphism showed similar age at onset, and these tended to be different from the ll genotype. The association between the 5-HTTLPR genotype and age at onset did not differ significantly among the three genotypes $(p=0.705)$ (Table 5$)$. We found a significant effect of the interaction between BDNF Val66Met and 5-HTTLPR and the age at onset of depression (Table 6). The positive result in the total patient group $(\mathrm{F}=2.808, \mathrm{df}=5$, $\mathrm{p}=0.018$ ) (Table 6), and in the female group $(\mathrm{F}=2.379, \mathrm{df}=5$, $\mathrm{p}=0.042$, data not shown), but not in the male patient group $(\mathrm{F}=1.413, \mathrm{df}=5, \mathrm{p}=0.245$, data not shown $)$ were revealed.

\section{DISCUSSION}

As in many studies, a genetic association between 5-HTTLPR and depressive disorder was found in this Korean sample. The s allele frequency in control group was $77.52 \%$, simi- lar to other studies on Korean population. ${ }^{26,27}$ We found a deviation from the Hardy-Weinberg equilibrium for 5-HTTLPR in the total and female patient subgroup but not in the male subgroup (Table 3 ). This result could add more support for the positive association between 5-HTTLPR and depressive disorder, along with the chi-square test for the allele and genotype association, although many factors influence HardyWeinberg equilibrium. This association was not found in the male subgroup. It is probably because of the small number of subjects. We included a relatively large number of control subjects for the comparison to overcome the statistical weakness based on the small number of patients in this study. Another reason could be a gender difference in genetic modulation of depression by 5-HTTLPR. A gender-specific genetic effect has been reported for 5-HTTLPR in many psychiatric phenotypes, particularly in terms of the interaction with an environmental effect. ${ }^{28,29}$ It is possible that the genetic effect is moderated in a gender-specific way. However, considering the limited statistical power of this study, further studies with a larger sample size are necessary.

BDNF Val66Met did not show an association with depressive disorder, and the interaction between BDNF Val66Met and 5-HTTLPR was found not to be associated with the diagnosis of depressive disorder in this study. The A allele (Met) frequency was $46.66 \%$ in this control group, not significantly different from other studies on Korean population. ${ }^{26,27,30}$ There was no deviation from Hardy-Weinberg equilibrium for BDNF Val66Met in our sample (Table 4). Despite much bio-

Table 3. Genetic association between BDNF Val66Met and depression

\begin{tabular}{|c|c|c|c|c|c|c|c|c|c|c|c|c|c|c|c|}
\hline & \multicolumn{5}{|c|}{ Total } & \multicolumn{5}{|c|}{ Male } & \multicolumn{5}{|c|}{ Female } \\
\hline & \multicolumn{2}{|c|}{ Allele } & \multicolumn{3}{|c|}{ Genotype } & \multicolumn{2}{|c|}{ Allele } & \multicolumn{3}{|c|}{ Genotype } & \multicolumn{2}{|c|}{ Allele } & \multicolumn{3}{|c|}{ Genotype } \\
\hline & 1 & 2 & 11 & 12 & 22 & 1 & 2 & 11 & 12 & 22 & 1 & 2 & 11 & 12 & 22 \\
\hline \multicolumn{16}{|l|}{ Frequency } \\
\hline Depression & 172 & 200 & 43 & 86 & 57 & 40 & 38 & 10 & 20 & 9 & 132 & 162 & 33 & 66 & 48 \\
\hline Control & 963 & 1101 & 218 & 527 & 287 & 212 & 226 & 54 & 104 & 61 & 751 & 875 & 164 & 423 & 226 \\
\hline \multicolumn{16}{|l|}{ HWE } \\
\hline Depression & \multicolumn{5}{|c|}{$\chi^{2}=0.911, p$-value $=0.634$} & \multicolumn{5}{|c|}{$\chi^{2}=0.027, p-$ value $=0.987$} & \multicolumn{5}{|c|}{$\chi^{2}=1.260, p-$ value $=0.533$} \\
\hline Control & \multicolumn{5}{|c|}{$\chi^{2}=0.692, p-$ value $=0.707$} & \multicolumn{5}{|c|}{$\chi^{2}=0.531, \mathrm{p}$-value $=0.767$} & \multicolumn{5}{|c|}{$\chi^{2}=1.771, \mathrm{p}$-value $=0.412$} \\
\hline \multicolumn{16}{|l|}{ Diagnosis of depression } \\
\hline$x^{2}$ & \multicolumn{2}{|c|}{0.022} & \multicolumn{3}{|c|}{1.472} & \multicolumn{2}{|c|}{0.220} & \multicolumn{3}{|c|}{0.401} & \multicolumn{2}{|c|}{0.167} & \multicolumn{3}{|c|}{2.583} \\
\hline $\mathrm{p}$-value & \multicolumn{2}{|c|}{0.881} & \multicolumn{3}{|c|}{0.479} & \multicolumn{2}{|c|}{0.639} & \multicolumn{3}{|c|}{0.818} & \multicolumn{2}{|c|}{0.683} & \multicolumn{3}{|c|}{0.275} \\
\hline Permutation p-value & \multicolumn{2}{|c|}{0.863} & \multicolumn{3}{|c|}{0.489} & \multicolumn{2}{|c|}{0.642} & & 0.826 & & & & & 0.268 & \\
\hline Age at onset & & & & & & & & & & & & & & & \\
\hline$\chi^{2}$ & & 657 & & 2.493 & & & & & 3.530 & & & & & 2.792 & \\
\hline $\mathrm{p}$-value & & 103 & & 0.286 & & & & & 0.171 & & & & & 0.248 & \\
\hline Permutation p-value & & 108 & & 0.281 & & & & & 0.191 & & & & & 0.274 & \\
\hline
\end{tabular}

HWE: Hardy-Weinberg Equilibrium, Permutation p-value: p-value of permutation test in UNPHASED 3.0.13 (1000 times), BDNF: brain derived neurotrophic factor 
Table 4. Genetic association between 5-HTTLPR and depression

\begin{tabular}{|c|c|c|c|c|c|c|c|c|c|c|c|c|c|c|c|}
\hline & \multicolumn{5}{|c|}{ Total } & \multicolumn{5}{|c|}{ Male } & \multicolumn{5}{|c|}{ Female } \\
\hline & \multicolumn{2}{|c|}{ Allele } & \multicolumn{3}{|c|}{ Genotype } & \multicolumn{2}{|c|}{ Allele } & \multicolumn{3}{|c|}{ Genotype } & \multicolumn{2}{|c|}{ Allele } & \multicolumn{3}{|c|}{ Genotype } \\
\hline & 1 & 2 & 11 & 12 & 22 & 1 & 2 & 11 & 12 & 22 & 1 & 2 & 11 & 12 & 22 \\
\hline \multicolumn{16}{|l|}{ Frequency } \\
\hline Depression & 306 & 66 & 133 & 40 & 13 & 64 & 14 & 28 & 8 & 3 & 242 & 52 & 105 & 32 & 10 \\
\hline Control & 1600 & 464 & 636 & 328 & 68 & 357 & 81 & 149 & 59 & 11 & 1243 & 383 & 487 & 269 & 57 \\
\hline \multicolumn{16}{|l|}{ HWE } \\
\hline Depression & \multicolumn{5}{|c|}{$\chi^{2}=12.887, p$-value $=0.002$} & \multicolumn{5}{|c|}{$\chi^{2}=3.594, p$-value $=0.166$} & \multicolumn{5}{|c|}{$\chi^{2}=9.364, p$-value $=0.009$} \\
\hline Control & \multicolumn{5}{|c|}{$\chi^{2}=8.011, p$-value $=0.018$} & \multicolumn{5}{|c|}{$\chi^{2}=2.476, p$-value $=0.290$} & \multicolumn{5}{|c|}{$\chi^{2}=5.366, p$-value $=0.068$} \\
\hline \multicolumn{16}{|l|}{ Diagnosis of depression } \\
\hline$x^{2}$ & \multicolumn{2}{|c|}{4.327} & \multicolumn{3}{|c|}{8.413} & \multicolumn{2}{|c|}{0.013} & \multicolumn{3}{|c|}{1.022} & \multicolumn{2}{|c|}{5.130} & \multicolumn{3}{|c|}{8.177} \\
\hline p-value & \multicolumn{2}{|c|}{0.038} & \multicolumn{3}{|c|}{0.015} & \multicolumn{2}{|c|}{0.909} & \multicolumn{3}{|c|}{0.600} & \multicolumn{2}{|c|}{0.024} & \multicolumn{3}{|c|}{0.017} \\
\hline Permutation p-value & \multicolumn{2}{|c|}{0.050} & \multicolumn{3}{|c|}{0.015} & \multicolumn{2}{|c|}{0.884} & & 0.592 & & & & & 0.015 & \\
\hline Age at onset & & & & & & & & & & & & & & & \\
\hline$\chi^{2}$ & 0.2 & & & 0.714 & & 0.1 & & & 0.91 & & & & & 0.738 & \\
\hline $\mathrm{p}$-value & 0.6 & & & 0.700 & & 0.7 & & & 0.63 & & & & & 0.691 & \\
\hline Permutation p-value & 0.6 & & & 0.700 & & 0.7 & & & 0.630 & & & & & 0.691 & \\
\hline
\end{tabular}

HWE: Hardy-Weinberg Equilibrium, Permutation p-value: p-value of permutation test in UNPHASED 3.0.13 (1000 times)

Table 5. Age at onset for genotypes of BDNF Val66Met and 5-HTTLPR

\begin{tabular}{ccccccc}
\hline Gene & Genotype & $\mathrm{N}$ & Mean & SD & F & p-value \\
\hline BDNF Val66Met & Met/Met & 43 & 46.65 & 16.61 & 1.236 & 0.293 \\
& Met/Val & 86 & 44.16 & 14.82 & \\
5-HTTLPR & Val/Val & 57 & 41.81 & 14.98 & 0.350 & 0.705 \\
& ss & 133 & 44.13 & 15.84 & 14.13 & \\
& sl & 40 & 44.73 & 40.69 & 13.88 & \\
\hline
\end{tabular}

ANOVA test was performed. BDNF: brain derived neurotrophic factor, 5-HTTLRP: The serotonin transporter-linked polymorphic region

Table 6. Genetic interaction of 5-HTTLPR and BDNF Val66Met on the age at onset of depression

\begin{tabular}{|c|c|c|c|c|c|c|c|}
\hline & & \multicolumn{6}{|c|}{ 5-HTTLPR } \\
\hline & & \multicolumn{3}{|c|}{ ss \& sl } & \multicolumn{3}{|c|}{11} \\
\hline & & Number & Mean & $\mathrm{SD}$ & Number & Mean & $\mathrm{SD}$ \\
\hline \multirow[t]{3}{*}{ BDNF Val66Met } & AA (Met/Met) & 37 & 49.11 & 16.39 & 6 & 31.50 & 7.97 \\
\hline & AG (Met/Val) & 83 & 44.36 & 15.04 & 3 & 38.67 & 1.16 \\
\hline & GG (Val/Val) & 53 & 40.74 & 14.66 & 4 & 56.00 & 13.37 \\
\hline
\end{tabular}

ANOVA test was performed ( $\mathrm{F}=2.808, \mathrm{df}=5, \mathrm{p}=0.018)$. 5-HTTLPR: serotonin transporter-linked polymorphic region, s: short allele, l: long allele, BDNF: brain-derived neurotrophic factor, A: alanine, G: guanine, Met: methionine, Val: valine, SD: standard deviation

chemical evidence for the role of BDNF in depressive disorders from studies such as animal models of depression, blood samples, and brain tissue of patients with depression, previous genetic association studies have not been consistent with respect to the association between BDNF Val66Met and depressive disorder. Even for Korean population, positive association with poststroke depression and depression in women with mastectomy due to breast cancer ${ }^{26,27}$ as well as no associ- ation with major depression ${ }^{30}$ have been reported. Our findings do not support a conclusion that the BDNF gene plays a significant role in depressive disorder in Korean population.

A gene-gene interaction between 5-HTTLPR and the BDNF Val66Met polymorphism was found on the age at onset of depressive disorder. It is unclear which allele conferred a greater risk for depressive disorder in this study. The Met allele of BDNF Val66Met was associated with older age at onset 
of depressive disorder if the patient had either the 5-HTTLPR ss or sl genotype. However, the BDNF Val66Met Val allele was associated with older age at onset of depressive disorder in the patient group with the 11 5-HTTLPR genotype, indicating that the BDNF Val66Met polymorphism affects age at onset of depressive disorders insufficiently by itself, but instead interacts with 5-HTTLPR. Early onset depressive disorder is considered to be different from late onset depression in some aspects. Genetic attribution to the age at onset is most likely to exist and several genes, not only one gene may play roles in interaction.

Genetic epistasis between BDNF Val66Met and 5-HTTLPR has been investigated for various psychiatric phenotypes. 5-HTTLPR 11 carriers scored lower on neuroticism scales in the presence of the BDNF Val variant and higher in the presence of the BDNF Met variant. ${ }^{31}$ The 5-HTTLPR ss genotype had a negative impact on mental health after childhood abuse only in patients with the BDNF Val/Val genotype. ${ }^{6}$ A genegene interaction on conscientiousness in adolescence was found in BDNF Val66Met Met allele carriers, with participants with the 5-HTTLPR ss genotype having the lowest conscientiousness scores. ${ }^{32}$ These studies support that a 5-HTTLPR and BDNF Val66Met gene-gene interaction plays a role on the phenotypes related to mood disorders and personality. However, no specific combination of risk alleles has been consistently found. Thus, replication studies with a larger sample size are warranted. A positive influence of the interaction between 5-HTTLPR and BDNF Val66Met on age at onset of depressive disorder was found in the total sample and in the female patients but not in the male patients. It is possible that we could not detect the difference statistically because our male subgroup was too small. Another reason for this could be a gender difference in the nature of the genetic association. Many studies have reported a gender difference in the genetic association of BDNF Val66 Met ${ }^{17,33-35}$ and 5-HTTL$\mathrm{PR}^{36-38}$ with depression. Clinically, depressions are clearly different according to gender in many ways. More studies focused on gender specific gene-gene interaction will be necessary.

This study had the following limitations. The number of patients included in the study was insufficient and far fewer than the number of control subjects. This led to a statistical limitation in interpreting the results in greater depth. Additionally, the polymorphisms tested represented only one site for each gene: 5-HTTLPR and BDNF. Even though these are functional polymorphisms, it is difficult to say whether they represent the entire gene. Another statistical limitation came from testing of multiple hypotheses. We conducted analyses for the whole group and subgroups by gender as well as an allele and genotype comparison between patients and controls. None of the positive results survived the Bonferroni correc- tion for multiple testing. Interpretation of these results should take into account the fact that the $\mathrm{p}$-values presented in this study were not corrected for multiple testing even though the permutation tests were performed and our positive results still showed significant p-values on permutation tests. Genetic power calculation of our sample was done for each polymorphism. Considering the minor allele 1 as a risk allele for 5-HTTLPR and Met allele for BDNF Val66Met and a significance level of $\mathrm{p}=0.05$, our analysis had a power of $79.42 \%$ for 5-HTTLPR and 65.23\% for BDNF Val66Met under a dominant model (genotypic relative risk of $\mathrm{AA}$ and $\mathrm{Aa}=1.5$; disease prevalence $=0.10$ ) calculated using the Genetic power calculator (http://pngu.mgh.harvard.edu/ purcell/gpc/cc2.html). Population stratification due to possibly different underlying genetic backgrounds between patients and controls should be considered as one of the causes of false-positive results for this type of genetic association study. However, the subjects were all ethnically Korean; therefore, it is unlikely that population stratification occurred.

In conclusion, we found that 5-HTTLPR, but not BDNF Val66Met, contributed to the diagnosis of depressive disorder. An interaction between BDNF Val66Met and 5-HTTLPR affected the age at onset of depressive disorders. Our findings suggest that BDNF Val66Met and 5-HTTLPR are significantly associated with depressive disorders in a complex way, not only in the diagnosis of depressive disorder but also in various clinical manifestations such as age at onset. The extent of genetic contribution of BDNF Val66Met and 5-HTTLPR could be different between males and females. Understanding the interactions between BDNF and the serotonin transporter is crucial to obtain a more comprehensive view of the molecular pathways of mood regulation. Our results should be considered cautiously because of insufficient power based on a relatively small number of patients. Further studies on this issue should follow.

\section{Acknowledgments}

This work was supported by National Research Foundation of Korea Grant funded by the Korean Government (KRF-2008-531-E00045 and 2011-0003164). We really appreciate all the subjects participating in this study.

\section{REFERENCES}

1. Heils A, Teufel A, Petri S, Seemann M, Bengel D, Balling U, et al. Functional promoter and polyadenylation site mapping of the human serotonin (5-HT) transporter gene. J Neural Transm Gen Sect 1995;102:247254.

2. Heils A, Teufel A, Petri S, Stober G, Riederer P, Bengel D, et al. Allelic variation of human serotonin transporter gene expression. J Neurochem 1996;66:2621-2624.

3. Clarke H, Flint J, Attwood AS, Munafo MR. Association of the 5-HTTLPR genotype and unipolar depression: a meta-analysis. Psychol Med 2010;40:1767-1778. 
4. Taylor MJ, Sen S, Bhagwagar Z. Antidepressant response and the serotonin transporter gene-linked polymorphic region. Biol Psychiatry 2010; 68:536-543.

5. Caspi A, Sugden K, Moffitt TE, Taylor A, Craig IW, Harrington H, et al. Influence of life stress on depression: moderation by a polymorphism in the 5-HTT gene. Science 2003;301:386-389.

6. Grabe HJ, Lange M, Wolff B, Volzke H, Lucht M, Freyberger HJ, et al. Mental and physical distress is modulated by a polymorphism in the 5-HT transporter gene interacting with social stressors and chronic disease burden. Mol Psychiatry 2005;10:220-224.

7. Risch N, Herrell R, Lehner T, Liang KY, Eaves L, Hoh J, et al. Interaction between the serotonin transporter gene (5-HTTLPR), stressful life events, and risk of depression: a meta-analysis. JAMA 2009;301:24622471.

8. Karg K, Burmeister M, Shedden K, Sen S. The serotonin transporter promoter variant (5-HTTLPR), stress, and depression meta-analysis revisited: evidence of genetic moderation. Arch Gen Psychiatry 2011;68: 444-454.

9. Autry AE, Monteggia LM. Brain-derived neurotrophic factor and neuropsychiatric disorders. Pharmacol Rev 2012;64:238-258.

10. Duman RS. Pathophysiology of depression: the concept of synaptic plasticity. Eur psychiatry 2002;17(Suppl 3):306-310.

11. Lee BH, Kim YK. The roles of BDNF in the pathophysiology of major depression and in antidepressant treatment. Psychiatry Investig 2010; 7:231-235.

12. Schumacher J, Jamra RA, Becker T, Ohlraun S, Klopp N, Binder EB, et al. Evidence for a relationship between genetic variants at the brain-derived neurotrophic factor (BDNF) locus and major depression. Biol Psychiatry 2005;58:307-314.

13. Ribeiro L, Busnello JV, Cantor RM, Whelan F, Whittaker P, Deloukas P, et al. The brain-derived neurotrophic factor (Val66Met) polymorphism and depression in Mexican-Americans. Neuroreport 2007;18:12911293.

14. Frielingsdorf H, Bath KG, Soliman F, Difede J, Casey BJ, Lee FS. Variant brain-derived neurotrophic factor val66met endophenotypes: implications for posttraumatic stress disorder. Ann N Y Acad Sci 2010;1208: 150-157.

15. Chen L, Lawlor DA, Lewis SJ, Yuan W, Abdollahi MR, Timpson NJ, et al. Genetic association study of BDNF in depression: finding from two cohort studies and a meta-analysis. Am J Med Genet Part B Neuropsychiatr Genet 2008;147B:814-821.

16. Liu X, Xu Y, Jiang S, Cui D, Qian Y, Jiang K. Family-based association study between brain-derived neurotrophic factor gene and major depressive disorder of chinese descent. Psychiatry Res 2009;169:169-172.

17. Verhagen M, van der Meij A, van Deurzen PA, Janzing JG, AriasVasquez A, Buitelaar JK, et al. Meta-analysis of the BDNF Val66Met polymorphism in major depressive disorder: effects of gender and ethnicity. Mol Psychiatry 2010;15:260-271.

18. Gatt JM, Nemeroff CB, Dobson-Stone C, Paul RH, Bryant RA, Schofield PR, et al. Interactions between BDNF val66met polymorphism and early life stress predict brain and arousal pathways to syndromal depression and anxiety. Mol Psychiatry 2009;14:681-695.

19. Martinowich K, Lu B. Interaction between BDNF and serotonin: role in mood disorders. Neuropsychopharmacology 2008;33:73-83.

20. Conti AC, Cryan JF, Dalvi A, Lucki I, Blendy JA. cAMP response element-binding protein is essential for the upregulation of brain-derived neurotrophic factor transcription, but not the behavioral or endocrine responses to antidepressant drugs. J Neurosci 2002;22:3262-3268.

21. Pezawas L, Meyer-Lindenberg A, Goldman AL, Verchinski BA, Chen G, Kolachana BS, et al. Evidence of biologic epistasis between BDNF and SLC6A4 and implications for depression. Mol Psychiatry 2008; 13:709-716.

22. Rybakowski JK, Suwalska A, Skibinska M, Dmitrzak-Weglarz M,
Leszczynska-Rodziewicz A, Hauser J. Response to lithium prophylaxis: Interaction between serotonin transporter and BDNF genes. Am J Med Genet B Neuropsychiatr Genet 2007;144B:820-823.

23. Kaufman J, Yang BZ, Douglas-Palumberi H, Grasso D, Lipschitz D, Houshyar S, et al. Brain-derived neurotrophic factor-5-HTTLPR gene interactions and environmental modifiers of depression in children. Biol Psychiatry 2006;59:673-680.

24. Kim JM, Stewart R, Kim SW, Yang SJ, Shin IS, Kim YH, et al. Interactions between life stressors and susceptibility genes (5-HTTLPR and BDNF) on depression in Korean elders. Biol Psychiatry 2007;62:423428.

25. McGuigan FE, Ralston SH. Single nucleotide polymorphism detection: allelic discrimination using TaqMan. Psychiatr Genet 2002;12:133-136.

26. Kim JM, Kim SW, Stewart R, Kim SY, Shin IS, Park MH, et al. Serotonergic and BDNF genes associated with depression 1 week and 1 year after mastectomy for breast cancer. Psychosom Med 2012;74:8-15.

27. Kim JM, Stewart R, Bae KY, Kim SW, Kang HJ, Shin IS, et al. Serotonergic and BDNF genes and risk of depression after stroke. J Affect Disord 2012;136:833-840.

28. Everaerd D, Gerritsen L, Rijpkema M, Frodl T, van Oostrom I, Franke $\mathrm{B}$, et al. Sex modulates the interactive effect of the serotonin transporter gene polymorphism and childhood adversity on hippocampal volume. Neuropsychopharmacology 2012;37:1848-1855.

29. Lei X, Chen C, He Q, Chen C, Moyzis RK, Xue G, et al. Sex determines which section of the slc6a4 gene is linked to obsessive-compulsive symptoms in normal chinese college students. J Psychiatr Res 2012;46: 1153-1160.

30. Pae CU, Chiesa A, Porcelli S, Han C, Patkar AA, Lee SJ, et al. Influence of BDNF variants on diagnosis and response to treatment in patients with major depression, bipolar disorder and schizophrenia. Neuropsychobiology 2012;65:1-11.

31. Terracciano A, Tanaka T, Sutin AR, Deiana B, Balaci L, Sanna S, et al. BDNF Val66Met is associated with introversion and interacts with 5-HTTLPR to influence neuroticism. Neuropsychopharmacology 2010; 35:1083-1089.

32. Hiio K, Merenakk L, Nordquist N, Parik J, Oreland L, Veidebaum T, et al. Effects of serotonin transporter promoter and BDNF Val66Met genotype on personality traits in a population representative sample of adolescents. Psychiatr Genet 2011;21:261-264.

33. Lavebratt C, Aberg E, Sjoholm LK, Forsell Y. Variations in FKBP5 and $\mathrm{BDNF}$ genes are suggestively associated with depression in a Swedish population-based cohort. J Affect Disord 2010;125:249-255.

34. Suchanek R, Owczarek A, Kowalczyk M, Kucia K, Kowalski J. Association between C-281A and Val66Met functional polymorphisms of BDNF gene and risk of recurrent major depressive disorder in Polish population. J Mol Neurosci 2011;43:524-530.

35. van Oostrom I, Franke B, Rijpkema M, Gerritsen L, Arias-Vasquez A, Fernandez G, et al. Interaction between BDNF Val66Met and childhood stressful life events is associated to affective memory bias in men but not women. Biol Psychol 2012;89:214-219.

36. Baune BT, Hohoff C, Mortensen LS, Deckert J, Arolt V, Domschke K. Serotonin transporter polymorphism (5-HTTLPR) association with melancholic depression: a female specific effect? Depress Anxiety 2008; 25:920-925.

37. Brummett BH, Boyle SH, Siegler IC, Kuhn CM, Ashley-Koch A, Jonassaint $\mathrm{CR}$, et al. Effects of environmental stress and gender on associations among symptoms of depression and the serotonin transporter gene linked polymorphic region (5-HTTLPR). Behav Genet 2008;38: 34-43.

38. Uddin M, Koenen KC, de Los Santos R, Bakshis E, Aiello AE, Galea S. Gender differences in the genetic and environmental determinants of adolescent depression. Depress Anxiety 2010;27:658-666. 